\title{
THE TURBULENT RESPONSE TO TIDAL AND LIBRATION FORCING
}

\author{
B. Favier ${ }^{1}$, A. Grannan ${ }^{2}$, T. Le Reun ${ }^{1}$, J. Aurnou $^{2}$ and M. Le Bars ${ }^{1}$
}

\begin{abstract}
In conjunction with thermo-solutal convection, the turbulence generated in planetary liquid cores may be due to the role of boundary forcing through geophysically relevant mechanisms such as precession, libration and tidal forcing (Le Bars et al. 2015). In this paper, we discuss laboratory equatorial velocity measurements and selected high-resolution numerical simulations to show the generation of developed turbulence driven by longitudinal libration or tidal forcing. In both cases, the transition to saturated turbulence is driven by an elliptical instability that excites inertial modes of the system. We find striking similarities in both the transition to bulk turbulence and the enhanced zonal flow hinting at a generic fluid response independent of the forcing mechanism. We finally discuss the relevance of this work to the planetary regime and possible directions for future investigations.
\end{abstract}

\section{Introduction}

The fluid dynamics of planetary cores remain an outstanding challenge involving global rotation, buoyancy, magnetic field and small-scale turbulence. Most studies are focused on buoyancy-driven flows since both radiogenic and primordial heat can sustain a superadiabatic temperature profile. While this model has been very successful in reproducing key observations of the Earth's magnetic field (Roberts \& King 2013), its validity has been questioned for smaller bodies such as the Moon or Ganymede (Dwyer et al. 2011; Le Bars et al. 2011; Sarson et al. 1997).

An alternative model relies on the energy stored in the rotational motion of planets. The orbital and spin motions of celestial bodies are always perturbed by gravitational interactions with orbiting companions, which can lead to periodic perturbations of their shape or rotation rate. The relevance of these harmonic

\footnotetext{
${ }^{1}$ Aix Marseille Univ., CNRS, Centrale Marseille, IRPHE, Marseille, France e-mail: favier@irphe.univ-mrs.fr

2 Department of Earth, Planetary, and Space Sciences, University of California - Los Angeles, Los Angeles, CA, USA
} 
forcings to planetary fluid motion was first noted by Malkus (1963), and since then, a growing body of research has been devoted to understanding the motions driven by precession (Lin et al. 2015; Barker 2016b), tides (Favier et al. 2014; Barker 2016a) and libration (Cébron et al. 2012; Favier et al. 2015). More generally, the interaction between small-scale convective motions and large-scale harmonic forcing (Zahn 1989; Cébron et al. 2010; Ogilvie \& Lesur, 2012) is also of interest.

In the following, we present recent advances in experiments and numerical simulations of the elliptical instability driven by tides and longitudinal libration in ellipsoidal containers. We focus on a particular case where sustained turbulence is achieved at parameters accessible by both experiments and simulations. We finally discuss the similarity between both types of forcing and their relevance to the planetary regime.

\section{Model and methods}

We consider the motion of an incompressible fluid with constant kinematic viscosity enclosed inside an ellipsoidal container. The geometry of the container is given by $(x / a)^{2}+(y / b)^{2}+(z / c)^{2}=1$ and $\beta=\left(a^{2}-b^{2}\right) /\left(a^{2}+b^{2}\right)$ is the ellipticity. For the librational forcing, the ellipsoidal container is rotating around the vertical axis at a rate $\Omega_{\text {spin }}\left(1+\epsilon \cos \omega_{L} t\right)$ where $\epsilon$ is the strength of the forcing and $\omega_{L}$ is its frequency. For the tidal forcing, the container is rotating around the vertical axis at a constant rate $\Omega_{\text {spin }}$ whereas the deformation is rotating at a different rate $\Omega_{\text {orb }}$. The frequency of the forcing in the frame of reference rotating at $\Omega_{\text {spin }}$ is simply $f_{L}=\omega_{L} / \Omega_{\text {spin }}$ for the libration case and $f_{T}=2\left(1-\Omega_{\text {orb }} / \Omega_{\text {spin }}\right)$ for the tides case. In both cases, we define the Ekman number as $E=\nu /\left(\Omega_{\text {spin }} R^{2}\right)$ where $R=\sqrt{\left(a^{2}+b^{2}\right) / 2}$. The base flow driven by these forcings (see Cébron et al. (2012) for more details) can parametrically excite inertial modes of the cavity leading to the so-called elliptical instability (Kerswell 2002). The typical growth rate for this instability is given by a balance between the parametric forcing and viscous damping in the bulk and in the boundary layers (Le Dizès 2000; Cébron et al. 2012). While the linear phase of this instability is well-understood, the nonlinear saturation remains unexplained in the general case.

Our goal here is to explore the turbulent regime well beyond the marginal case. The experimental setups are shown in Figure 1. For the tidal forcing, we use a silicone spherical container rotating around the vertical axis. Two rollers, rotating around the container and along the vertical axis, apply an axial deformation leading to an ellipsoidal shape. For the libration forcing, a rigid ellipsoidal container is rotating around the vertical axis. A secondary oscillatory rotation, also along the vertical axis in the case of longitudinal libration, is applied by a secondary motor. In both cases, quantitative measurements are performed using Particle Image Velocimetry (PIV). More details concerning the experimental devices and measurements can be found in previous papers (Le Bars et al. 2010; Grannan et al. 2014; Grannan et al. 2016). 

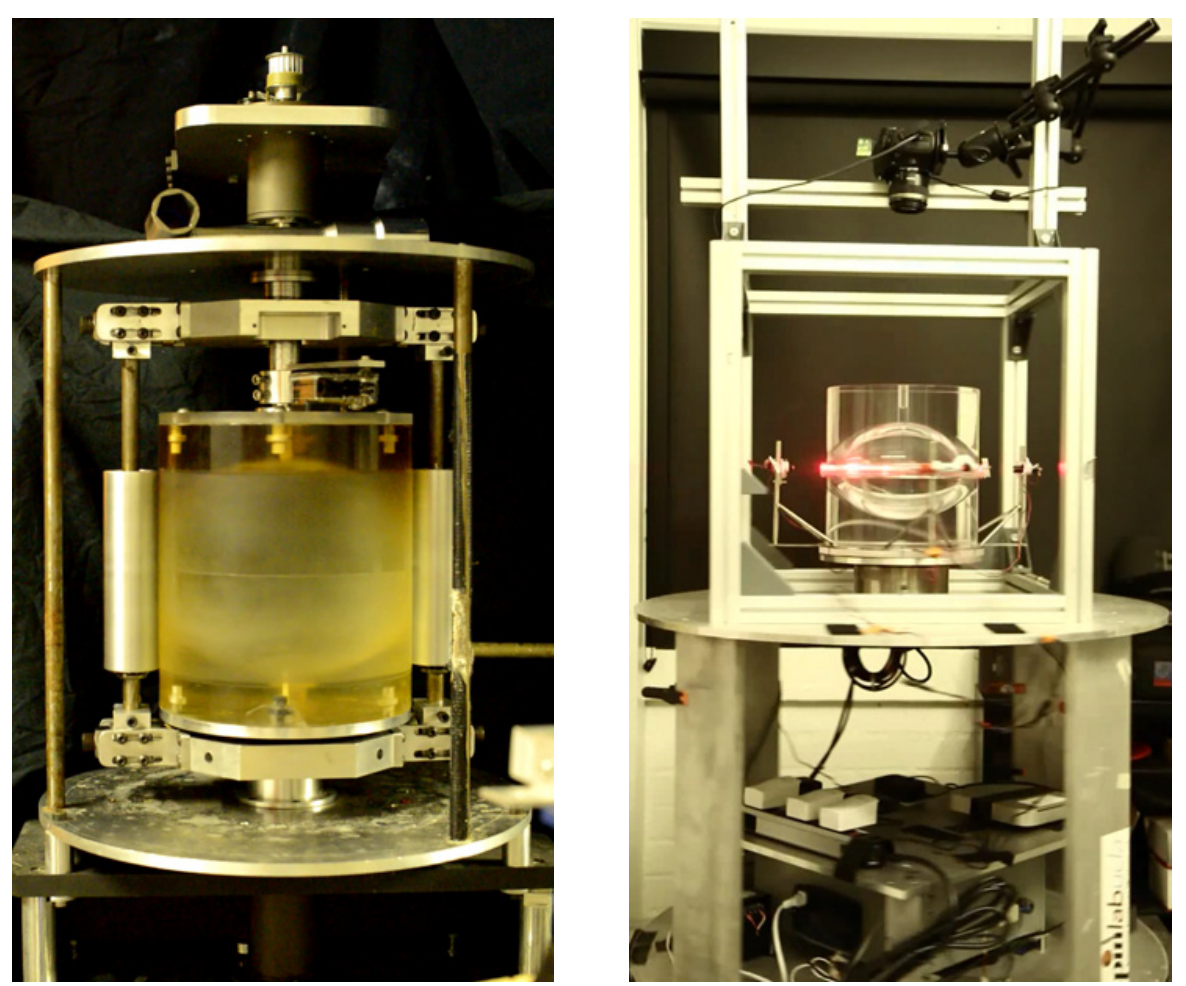

Fig. 1. Tidal experimental setup (left, IRPHE, Marseille) and longitudinal libration experimental setup (right, SpinLab, UCLA).

In addition to the experimental measurements, we also perform Direct Numerical Simulations (DNS). We use the spectral element solver Nek5000 (http:// nek5000.mcs.anl.gov) developed and supported by Paul Fischer and collaborators (Fischer et al. 2007). Spectral element methods have excellent convergence properties, required to simulate turbulent flows, while being able to consider complex geometries. In all cases, we solve for the flow in the frame of the elliptical deformation. In the case of tides, this implies that a steady tangential velocity is imposed at the boundary. In the case of libration, this implies that the Coriolis force has to be supplemented with a Poicaré force due the rate of change of the rotation frequency. More details about the numerical simulations can be found in Favier et al. (2015).

\section{Results}

In this article, we focus on a particular type of excitation corresponding to $f_{L}=$ $f_{T}=4$. We also fix the Ekman number to $E \approx 2 \times 10^{-5}$ for the experiment whereas $5 \times 10^{-5}<E<10^{-4}$ for the simulations. The effect of varying the 


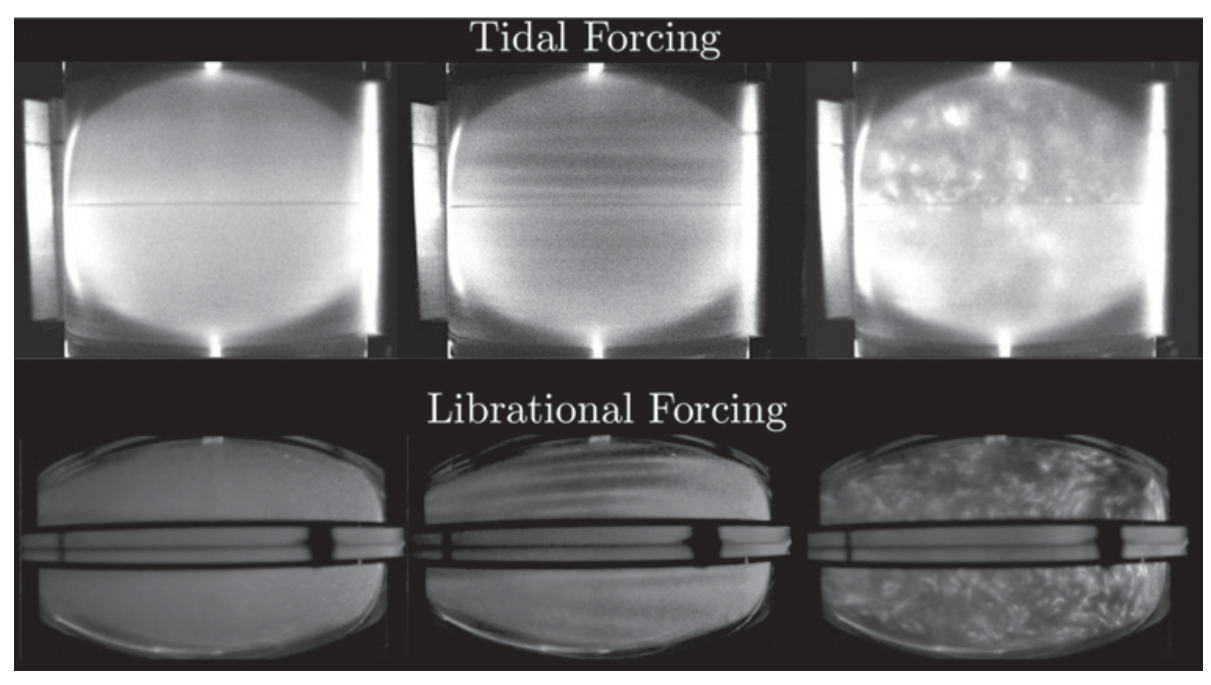

Fig. 2. Visualizations in a meridional plane using Kalliroscope for the tidal forcing (top) and longitudinal libration forcing (bottom). The forcing frequency is $f=4$ and the Ekman number is $E=2 \times 10^{-5}$ in both cases. Time is increasing from left to right.

Ekman number is discussed further in Favier et al. (2015). The amplitude of the libration is fixed to $\epsilon=0.8$ in a geometry with $\beta=0.34$ whereas the amplitude of the deformation is $\beta=0.09$ for the tidal setup.

Let us first discuss the experimental results. We show in Figure 2 visualizations of a meridional plane across the ellipsoid at different times. Initially, the forcing imposes an oscillating base flow with elliptic streamlines. This flow is unstable to the elliptical instability, which couples with two inertial modes of the cavity (which correspond here to the elongated structures observed in the middle of Fig. 2). After a linear phase characterized by an exponential growth of the modes, the flow collapses into bulk-filling turbulence. For this particular choice of parameters, the flow remains turbulent and quasi-stationary as long as the forcing is maintained. More details about the instability mechanism and mode couplings can be found in (Grannan et al. 2014; Favier et al. 2015).

This behavior is recovered numerically, where we observe first two inertial modes at half the frequency of the forcing, followed by a turbulent saturation (see Figs. 3-4). When varying the amplitude of the deformation in the tides configuration, we recover that the amplitude of the fluctuating flow scales linearly with it, as found in local models of the instability (Barker \& Lithwick 2013). Both numerically and experimentally, the similarity between the tidal and libration forcing is striking. This is further confirmed by looking at the temporal power spectra of horizontal velocity signals, both measured experimentally and extracted from the simulations (Fig. 5). In all cases, we clearly see a dominant peak at the forcing frequency $f=4$ and a second peak at half the forcing frequency corresponding 

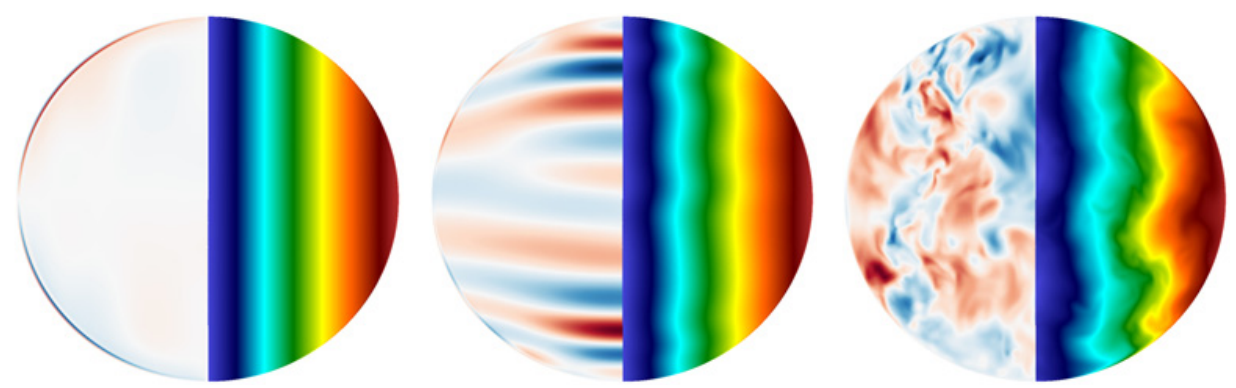

Fig. 3. Meridional slices along the long axis of the tidally-forced ellipsoid from DNS. We show the vertical velocity on the left part of each panel and the velocity amplitude on the right part. Time is increasing from left to right. The ellipticity is $\beta=0.09$, the Ekman number is $E=5 \times 10^{-5}$ and the tidal frequency is $f_{T}=4$.
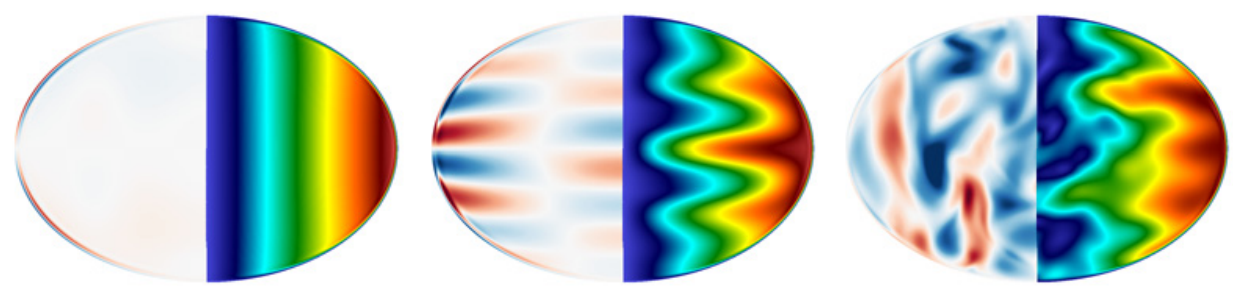

Fig. 4. Meridional slices along the long axis of the librating ellipsoid from DNS. We show the vertical velocity on the left part of each panel and the total velocity amplitude (including the base flow) on the right part. Time is increasing from left to right. The ellipticity is $\beta=0.34$, the Ekman number is $E=10^{-4}$, the libration frequency is $f_{L}=4$ and the librating amplitude is $\epsilon=0.8$.

to the primary parametric resonance. At these low Ekman numbers, the saturation of the instability generates two additional contributions: at low frequency, we observe additional peaks corresponding to triadic resonances between the primary inertial modes and other inertial modes of the cavity (Favier et al. 2015). At higher frequencies, we observe a cascade corresponding to small-scale turbulent fluctuations. These fluctuations are not inertial waves since their frequencies are larger than twice the spin rate, but correspond to small-scale turbulent fluctuations for which the Coriolis force is important, but not dominant.

\section{Discussion}

In this paper, we discussed several recent advances in the evidence for turbulent motions driven by the elliptical instability in laboratory experiments and numerical simulations. We showed that a sustained regime is possible, in addition to the intermittent regime observed both experimentally (Eloy et al. 2000) and 


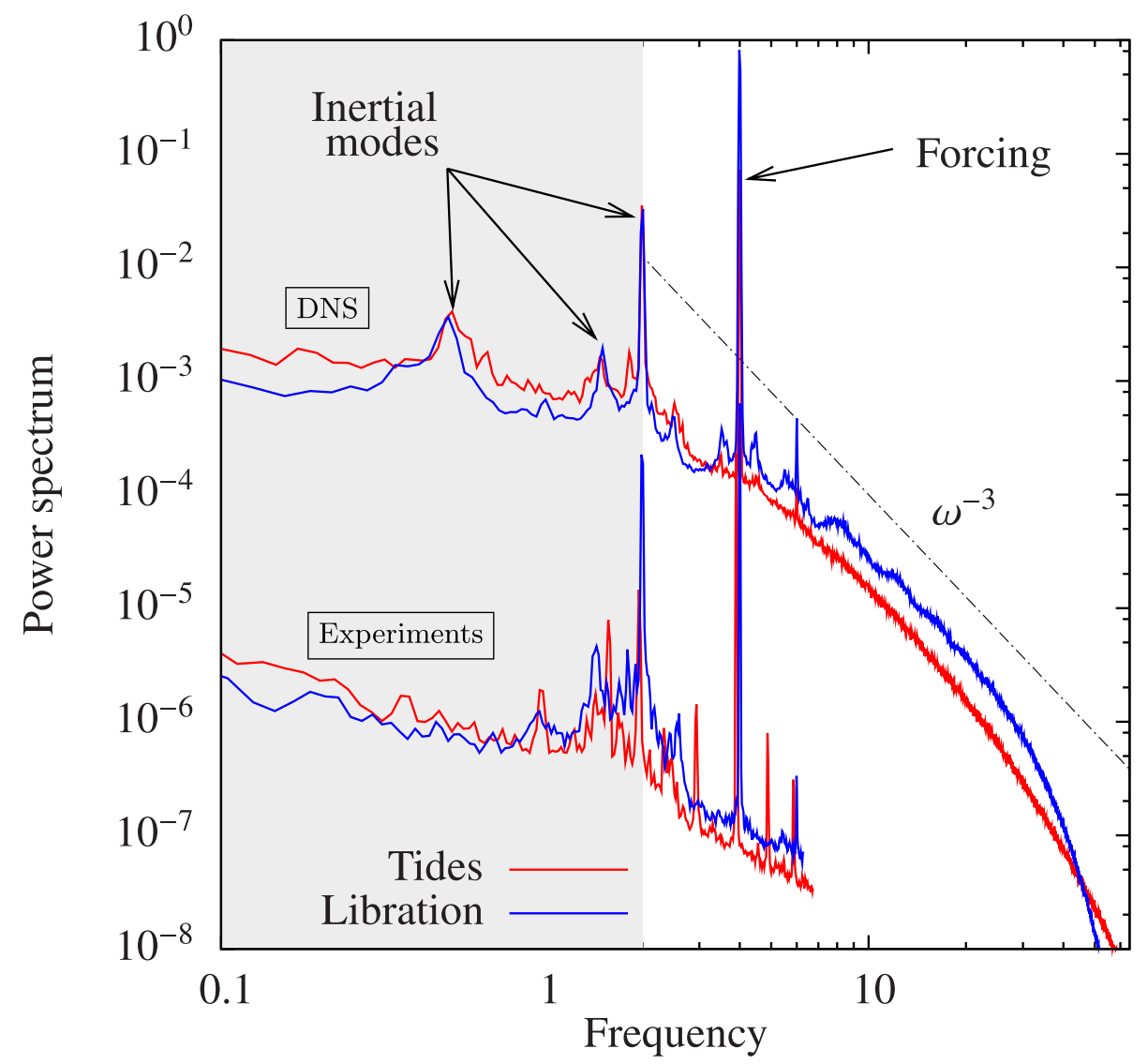

Fig. 5. Temporal power spectra of horiztonal velocity components for both libration and tides configurations. The experimental results are shifted down by three decades compared to the numerical results for clarity.

numerically (Barker \& Lithwick 2013). Additional details concerning the effect of changing the Ekman number, the deformation or the forcing frequency, and the generation of zonal flows can be found in (Cébron et al. 2012; Grannan et al. 2014; Favier et al. 2015).

Let us now discuss the relevance of these results to the planetary regime. As shown in Figure 6, the parameter space covered by experiments and simulations is far from the geophysically relevant regime. Experimentally and numerically, we can only achieve a regime with large viscous damping (high Ekman numbers) and therefore strong forcing amplitudes (in order to remain above the instability threshold). In planetary setting, the forcing is much weaker but so is the viscous damping, so that the instability is still expected for some close-in planets (Cébron et al. 2012). Similarly to convection studies, extrapolation from a regime 


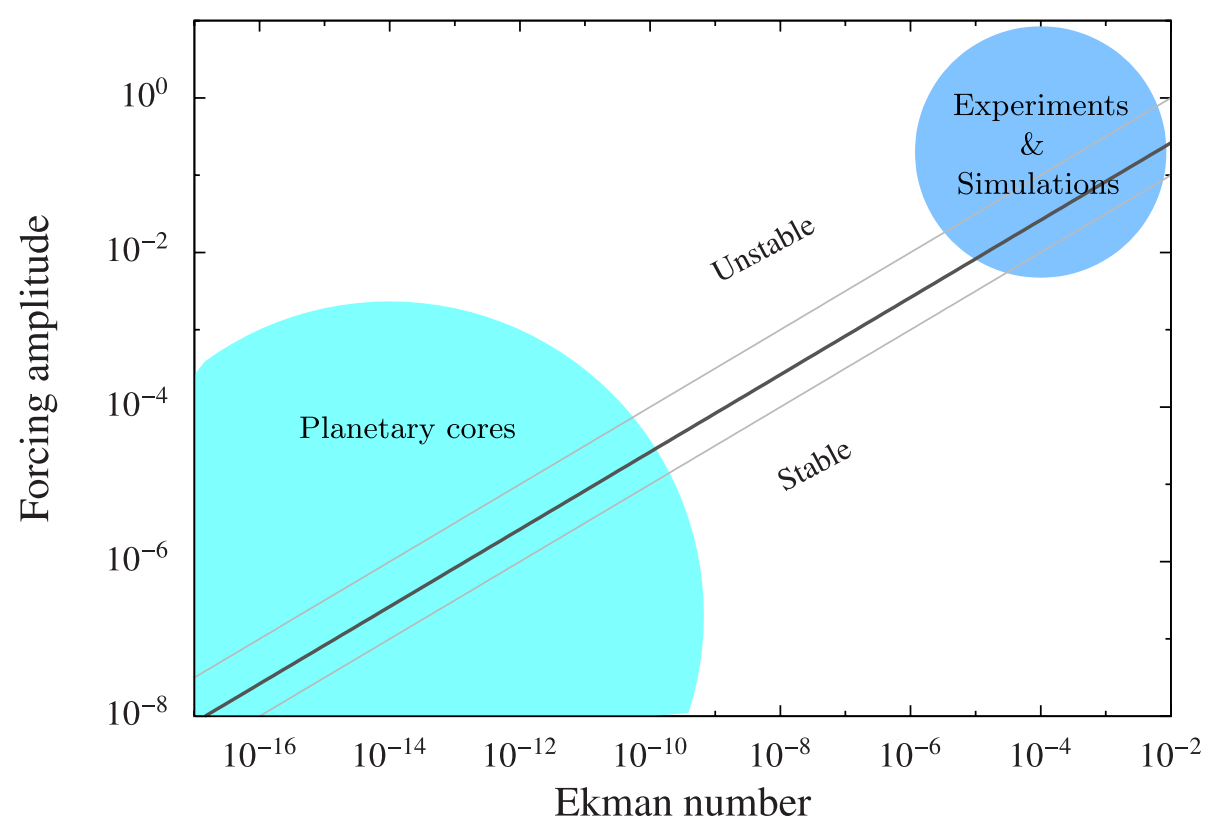

Fig. 6. Schematic of the parameter regimes accessible numerically and in the laboratory compared to the geophysically relevant regime. The solid black line corresponds to the instability threshold taking into account viscous damping inside boundary layers.

accessible to numerical simulations is therefore required. This remains a tremendous challenge since reducing the Ekman number in experiments requires increasing the size of the container or the rotation rate, both leading to technical difficulties. Numerically, the only hope is probably coming from local models of the instability (Barker \& Lithwick 2013). Despite neglecting the global geometry and viscous boundary layers, these models might help us in the future reaching a regime closer to planets. In addition, we have so far neglected the possible presence of an inner core. The nature of inertial modes in spherical shells is drastically modified which could in turn affect the instability mechanism discussed here. Finally, the coupling between these harmonic forcings and thermal convection, with or without inertial instabilities, remains a tremendous challenge.

This work was partly funded by the French Agence Nationale pour la Recherche (Grant No. ANR-13-JS05-0004-01). B. Favier would like to acknowledge support from IDRIS (Institut du Développement et des Ressources en Informatique Scientifique) for computational time on Turing (projects number 100508 and 100614) and from the HPC resources of Aix-Marseille Université financed by the project Equip@Meso (ANR-10-EQPX-2901) of the program Investissements d'Avenir supervised by the Agence Nationale pour la Recherche (project 15B011). A. Grannan and J. Aurnou gratefully acknowledge financial support of the National Science Foundation (NSF) Geophysics Program. 


\section{References}

Barker, A.J., 2016a, MNRAS, 459(1), 939

Barker, A.J., 2016b, MNRAS, 460(3), 2339

Barker, A.J., \& Lithwick, Y., 2013, MNRAS, 435, 3614

Cébron, D., Le Bars, M., Moutou, C., \& Le Gal, P., 2012, Astro. Astrophys., 539, A78

Cébron, D., Le Bars, M., Noir, J., \& Aurnou, J.M., 2012, Phys. Fluids, 24(6), 061703

Cébron, D., Maubert, P., \& Le Bars, M., 2010, Geophys. J. Int., 182, 1311

Dwyer, C.A., Stevenson, D.J., \& Nimmo, F., 2011, Nature, 479, 212

Eloy, C., Le Gal, P., \& Le Dizès, S., 2000, Phys. Rev. Lett., p. 3400

Favier, B., Barker, A.J., Baruteau, C., \& Ogilvie, G.I., 2014, MNRAS, 439, 845

Favier, B., Grannan, A.M., Le Bars, M., \& Aurnou, J.M., 2015, Phys. Fluids, 27

Fischer, P.F., Loth, F., Lee, S., Lee, S., Smith, D., \& Bassiouny, H., 2007, Comput. Meth. Appl. Mech. Eng., 196, 3049

Grannan, A.M., Favier, B., Le Bars, M., \& Aurnou, J.M., 2016, Geophys. J. Int., accepted

Grannan, A.M., Le Bars, M., Cébron, D., \& Aurnou, J.M., 2014, Phys. Fluids, 26(12), 126601

Kerswell, R.R., 2002, Annu. Rev. Fluid Mech., 34, 83

Le Bars, M., Cébron, D., \& Le Gal, P., 2015, Annu. Rev. Fluid Mech., 47, 163

Le Bars, M., Lacaze, L., Le Dizès, S., Le Gal, P., \& Rieutord, M., 2010, Phys. Earth Planet. Interiors, 178,48

Le Bars, M., Wieczorek, M.A., Karatekin, Ö., Cébron, D., \& Laneuville, M., 2011, Nature, 479, 215

Le Dizès, S., 2000, Phys. Fluids, 12, 2762

Lin, Y., Marti, P., \& Noir, J., 2015, Phys. Fluids, 27(4)

Malkus, W.V.R., 1963, J. Geophys. Res., 68(10), 2871

Ogilvie, G.I., \& Lesur, G., 2012, MNRAS, 422, 1975

Roberts, P.H., \& King, E.M., 2013, Rep. Prog. Phys., 76(9), 096801

Sarson, G.R., Jones, C.A., Zhang, K., \& Schubert, G., 1997, Science, 276(5315), 1106

Zahn, J.-P., 1989, Astron. Astrophys., 220, 112 